\title{
Production-inventory games: A new class of totally balanced combinatorial optimization games
}

\author{
Luis A. Guardiola ${ }^{\mathrm{a}}$, Ana Meca ${ }^{\mathrm{a}}$, Justo Puerto ${ }^{\mathrm{b}, *}$ \\ a Operations Research Center, Universidad Miguel Hernández, Avda. de la Universidad s/n, \\ Elche, 03202 Alicante, Spain \\ ${ }^{\mathrm{b}}$ Facultad de Matemáticas, Universidad de Sevilla, 41012 Sevilla, Spain
}

Received 18 August 2004

\begin{abstract}
In this paper we introduce a new class of cooperative games that arise from production-inventory problems. Several agents have to cover their demand over a finite time horizon and shortages are allowed. Each agent has its own unit production, inventory-holding and backlogging cost. Cooperation among agents is given by sharing production processes and warehouse facilities: agents in a coalition produce with the cheapest production cost and store with the cheapest inventory cost. We prove that the resulting cooperative game is totally balanced and the Owen set reduces to a singleton: the Owen point. Based on this type of allocation we find a population monotonic allocation scheme for this class of games. Finally, we point out the relationship of the Owen point with other well-known allocation rules such as the nucleolus and the Shapley value.
\end{abstract}

(c) 2007 Elsevier Inc. All rights reserved.

JEL classification: $\mathrm{C} 71$

Keywords: Production-inventory games; Totally balanced combinatorial optimization games; Core-allocations;

Owen-allocations; Monotonicity rules

\section{Introduction}

One of the main objectives of management of firms is cost reduction. In order to achieve this goal, groups of firms might form coalitions to diminish operation costs making dynamic

\footnotetext{
* Corresponding author. Fax: +34 954622800.

E-mail addresses: ana.meca@umh.es (A. Meca), puerto@us.es (J. Puerto).
} 
decisions throughout a finite planning horizon. In tactical planning of enterprises which produce indivisible goods, operation costs mainly consist of production, inventory-holding, and backlogging costs. These coalitions should induce individual and collective cost reductions; thus, stability is achieved in the process of enterprise cooperation.

In our framework a coalition allows each of its members of have access to the technologies owned by the other members of the coalition. Thus, members of a coalition can use the lowestcost technology of the firms in the coalition. Planning is done throughout a finite time horizon; therefore, at the beginning of each period, the costs to the members of a coalition, which depend on the best technology at that point, may change.

The model that represents that situation is the dynamic, discrete, finite planning horizon production-inventory problem with backlogging. The objective of any group of firms is to satisfy the demand for indivisible goods in each period at a minimum cost. This is a well-known combinatorial optimization problem for which the algorithm by Wagner and Whitin (1958) provides optimal solutions by dynamic programming techniques. The optimal solutions of this problem lead to the best production-inventory policy for the group of firms. These policies generate an optimal operation cost for the entire group. The question is what portion of this cost is to be supported by each firm. Cooperative game theory provides the natural tools for answering this question.

The analysis of inventory situations is not new. Thus, one can find in the literature several centralization inventory models approached from this point of view. The interested reader is referred to Eppen (1979), Kohli and Park (1989), Gerchak and Gupta (1991), Robinson (1993), Hartman and Dror (2003, 2005, 1996), Hartman et al. (2000), Anupindi et al. (2001), Müller et al. (2002), Meca et al. (2003, 2004), Meca (2007), Minner (2007), Tijs et al. (2005) and Slikker et al. (2005) among others, for comprehensive literature on this subject. Other operations research games are studied as well. For a clear and detailed presentation of operations research games (including inventory games) we refer to Borm et al. (2001). We are not aware of any reference in the literature of centralization in inventory models that analyzes inventory models for which optimal operation is only defined implicitly as the optimal solution of a combinatorial problem (as it is the case in the discrete review model in this paper). In this regards, our approach makes a step forward.

The study of cooperative combinatorial optimization games, which are defined through characteristic functions given as optimal values of combinatorial optimization problems, is a fruitful topic (see, for instance, Shapley and Shubik, 1972; Dubey and Shapley, 1984; Granot, 1986; Tamir, 1992; Deng et al., 1999, 2000; Faigle and Kern, 2000). There are characterizations of the total balancedness of several classes of these games. Inventory games and combinatorial optimization games are up to date disjoint classes of games. While in the former class there is always an explicit form for the characteristic function of each game, the characteristic function of the games in the latter class it is defined implicitly as the optimal value of an optimization problem in integer variables.

In this paper we introduce a class of production-inventory games which combine the characteristics of inventory and combinatorial optimization games: this class models cooperation on production and storage of indivisible goods and its characteristic function is defined implicitly as the optimal value of a combinatorial optimization problem. It turns out to be a new class of totally balanced combinatorial optimization games.

We start by introducing definitions and notations in Section 2. In Section 3 we give a complete description of the production-inventory problem (PI-problem). A natural variant of this problem is addressed in Section 4. Several agents, each one facing a PI-problem, decide to cooperate to 
reduce costs, and then a production-inventory situation (PI-situation) arises. Then, for each PIsituation, the corresponding cooperative game structure, namely production-inventory game (PIgame), is defined. The main results (total balancedness and an explicit form for the characteristic function) are stated in this section. Section 5 completes the study of PI-games by showing that the Owen set of a PI-situation (the set of allocations that are achievable through dual solutions) shrinks to a singleton: the Owen point. Its explicit form is also provided, and moreover, it is proved that the Owen point can be reached through a population monotonic allocation scheme. In addition, a necessary and sufficient condition for the core of a PI-game to be a singleton: the Owen point, is presented. We propose the Owen point as a core-allocation for a PI-game which is easy to calculate and satisfies good properties. Finally, we point out the relationship of the Owen point with some well-known point solutions in cooperative game theory.

\section{Preliminaries}

Production-inventory games constitute a class of cooperative cost games with transferable utility (TU games). A TU cost game is a pair $(N, c)$, where $N=\{1,2, \ldots, n\}$ is the finite player set and $c: \mathcal{P}(N) \rightarrow \mathbb{R}$ the characteristic function satisfying $c(\emptyset)=0$. The subgame related to coalition $S, c_{S}$, is the restriction of the mapping $c$ to the subcoalitions of $S$. We denote by lower case letter $s$ the cardinality of set $S$, i.e. $\operatorname{card}(S)=s$, for all $S \subseteq N$. A cost-sharing vector will be $x \in \mathbb{R}^{n}$ and, for every coalition $S \subseteq N$ we shall write $x(S):=\sum_{i \in S} x_{i}$ the cost-sharing to coalition $S$ (where $x(\emptyset)=0$ ). The core of the game $(N, c)$ consists of those cost-sharing vectors which allocate the cost of the grand coalition in such a way that every other coalition pays at most its cost, given by the characteristic function: Core $(N, c)=\left\{x \in \mathbb{R}^{n} / x(N)=c(N)\right.$ and $x(S) \leqslant c(S)$ for all $S \subset N\}$. In the following, cost-sharing vectors belonging to the core will be called core-allocations. A cost game $(N, c)$ has a nonempty core if and only if it is balanced (see Bondareva, 1963; Shapley, 1967). It is a totally balanced game if the core of every subgame is nonempty. Totally balanced games were introduced by Shapley and Shubik in the study of market games (see Shapley and Shubik, 1969).

A population monotonic allocation scheme (see Sprumont, 1990), or pmas, for the game $(N, c)$ is a collection of vectors $y^{S} \in \mathbb{R}^{S}$ for all $S \subseteq N, S \neq \emptyset$ such that $y^{S}(S)=c(S)$ for all $S \subseteq N, S \neq \emptyset$, and $y_{i}^{S} \geqslant y_{i}^{T}$ for all $S \subseteq T \subseteq N$ and $i \in S$. Note that if $\left(y^{S}\right)_{\emptyset \neq S \subseteq N}$ is a pmas for $(N, c)$, then $y^{S} \in \operatorname{Core}\left(N, c_{S}\right)$ for all $S \subseteq N, S \neq \emptyset$. Hence, the set of payoff vectors that can be reached through a pmas can be seen as a refinement of the core. Every cost game with pmas is totally balanced. However, it is not possible to get a pmas with any random selection of cost-sharings and there are totally balanced cost games without pmas. A core-allocation for $(N, c)$, denoted by $x \in \operatorname{Core}(N, c)$, is reached through a pmas if there exists $\left(y^{S}\right) \emptyset \neq S \subseteq N$ for the game $(N, c)$ such that $y_{i}^{N}=x_{i}$ for all $i \in N$.

A game is said to be subadditive when for all disjoint coalitions $S$ and $T, c(S \cup T) \leqslant c(S)+$ $c(T)$ holds. In a subadditive game, it will always be beneficial for two disjoint coalitions to cooperate and form a larger coalition. Balanced cost games might not be subadditive but they always satisfy subadditive inequalities involving the grand coalition. However, totally balanced cost games are subadditive. A well-known class of balanced and subadditive games is the class of concave games (see Shapley, 1971).

Finally to simplify the presentation, for a generic problem $(P)$ we will denote in the following by $\operatorname{val}(P), F(P)$ and $O(P)$, the optimal value, the set of feasible solutions and the set of optimal solutions of problem $(P)$, respectively. 


\section{Production-inventory problems}

To start with, the basic form of the production-inventory model is now described (the interested reader is referred to Johnson and Montgomery, 1974 for further details). Demand for a single product occurs during each of $T$ consecutive time periods that are numbered 1 through $T$. The demand that occurs during a given period can be satisfied by production during that period, during any earlier period (as inventory is carried forward) or can be backlogged to be covered by production at future periods (as backlogged demand is accumulated). Inventory at period 1 is zero, and inventory at the end of period $T$ is required to be zero. The model includes production, inventory and backlogging costs. The objective is to schedule production so as to satisfy demand at minimum cost. Formally, a production-inventory problem (PI-problem for short) is a 5-tuple $(T, d, h, b, p)$ where:

- $T$ is the planning horizon.

- $d=\left(d_{1}, \ldots, d_{T}\right) \geqslant 0, d_{t}=$ demand during period $t, t=1, \ldots, T$.

- $h=\left(h_{1}, \ldots, h_{T}\right) \geqslant 0, h_{t}=$ unit inventory carrying costs in period $t, t=1, \ldots, T$.

- $b=\left(b_{1}, \ldots, b_{T}\right) \geqslant 0, b_{t}=$ unit backlogging carrying costs in period $t, t=1, \ldots, T$.

- $p=\left(p_{1}, \ldots, p_{T}\right) \geqslant 0, p_{t}=$ unit production costs in period $t, t=1, \ldots, T$.

The decision variables of the model are:

- $q_{t}=$ production during period $t$.

- $I_{t}=$ inventory at hand at the end of period $t$.

- $E_{t}=$ backlogged demand at the end of period $t$.

The reader may notice that $I$ and $E$ variables are instrumental and help to clarify presentation while $q$ is the only actual set of decision variables. (Once $q$ is known the others can be obtained solving a system of linear equations.) These decision variables are required to be in integer quantities. The resolution amounts to the following mathematical programming formulation:

$$
\begin{array}{ll}
\min & \sum_{t=1}^{T}\left(p_{t} q_{t}+h_{t} I_{t}+b_{t} E_{t}\right) \\
\text { s.t. } & I_{0}=I_{T}=E_{0}=E_{T}=0, \\
& I_{t}-E_{t}=I_{t-1}-E_{t-1}+q_{t}-d_{t}, \quad t=1, \ldots, T, \\
& q_{t}, I_{t}, E_{t}, \quad \text { nonnegative, integer, } t=1 \ldots, T .
\end{array}
$$

Constraint (1) ensures initial and final conditions on inventory and backlogged demand. Matter is conserved, and (2) requires that the sum of the inventory at the end of a period is the sum of the inventory at the end of previous period minus consumption. Finally, constraint (3) ensures nonnegativity and integrality of the variables in the problem.

We associate with problem (PI) its linear relaxation (LPI). This problem turns out to be

$$
\begin{array}{ll}
\min & \sum_{t=1}^{T}\left(p_{t} q_{t}+h_{t} I_{t}+b_{t} E_{t}\right) \\
\text { s.t. } & I_{0}=I_{T}=E_{0}=E_{T}=0, \\
& I_{t}-E_{t}=I_{t-1}-E_{t-1}+q_{t}-d_{t}, \quad t=1, \ldots, T, \\
& q_{t} \geqslant 0, I_{t} \geqslant 0, E_{t} \geqslant 0, \quad t=1, \ldots, T .
\end{array}
$$


First of all, we note in passing that if vector $d$ has integer components all the extreme points of the feasible region of (LPI) are integers. Indeed, the constraint matrix of problem (LPI) is a 0 , \pm 1 valued matrix where each column has at most one +1 and at most one -1 , then it is totally unimodular and the result is well known (see, e.g., Garfinkel and Nemhauser, 1972).

Let (DLPI) be the dual of (LPI). This problem is given as

$$
\begin{aligned}
\text { (DLPI) } \quad \max & \sum_{t=1}^{T} y_{t} d_{t} \\
\text { s.t. } \quad & y_{t} \leqslant p_{t}, \quad t=1, \ldots, T, \\
& y_{t+1}-y_{t} \leqslant h_{t}, \quad t=1, \ldots, T-1, \\
& -y_{t+1}+y_{t} \leqslant b_{t}, \quad t=1, \ldots, T-1 .
\end{aligned}
$$

The consequence of the above discussion is that

$$
\operatorname{val}(\mathrm{PI})=\operatorname{val}(\mathrm{LPI})=\operatorname{val}(\mathrm{DLPI}) .
$$

Moreover, problems (PI) and (LPI) have integer optimal solutions.

Let

$$
\begin{aligned}
h_{k t} & =\sum_{r=k}^{t-1} h_{r} \quad \text { for any } k<t, t=2, \ldots, T, h_{k 1}=0, k<1, \\
b_{t k} & =\sum_{r=t}^{k-1} b_{r}, \quad \text { for any } k>t, t=1, \ldots, T-1, b_{T k}=0, k>T .
\end{aligned}
$$

According to this notation one can check that a feasible solution for (DLPI) is given as

$$
y_{t}^{*}=\min \left\{p_{t}, \min _{k<t}\left\{p_{k}+h_{k t}\right\}, \min _{k>t}\left\{p_{k}+b_{t k}\right\}\right\}, \quad t=1, \ldots, T,
$$

where

$$
p_{k}= \begin{cases}p_{1} & \text { if } k<1, \\ p_{T} & \text { if } k>T .\end{cases}
$$

The reader may notice that this solution induces a feasible solution in the primal problem. In this solution the demand $d_{t}$ that occurs in period $t$ is produced according to the following scheme:

$$
\begin{cases}\text { in period } t & \text { if } y_{t}^{*}=p_{t}, \\ \text { in period } k_{h} & \text { if } y_{t}^{*}=p_{k_{h}}+h_{k_{h}}, k_{h}<t \\ \text { in period } k_{b} & \text { if } y_{t}^{*}=p_{k_{b}}+b_{t k_{b}}, k_{b}>t\end{cases}
$$

Lemma 3.1. The vector $y^{*}$ given in (13) is an optimal solution of problem (DLPI).

Proof. The reader can easily check that $y^{*}$ satisfies all the constraints of problem (DLPI). This solution induces a feasible production plan $\left(q^{*}, I^{*}, E^{*}\right)$ in the primal problem (PI) so that $\sum_{t=1}^{T}\left(p_{t} q_{t}^{*}+h_{t} I_{t}^{*}+b_{t} E_{t}^{*}\right)=\sum_{t=1}^{T} d_{t} y_{t}^{*}$. Therefore, both solutions are optimal in their corresponding problems.

The reader may notice that if it were assumed that all the unit costs $h, b$ and $p$ are nonnegative integers then the solution $y^{*}$ would also be an integer. Moreover, we would like to have more 
information about the structure of the optimal solution set to (DLPI). The next results show that the optimal solution $y^{*}$ given by (13) is the componentwise upper bound of any optimal solution of (DLPI). Hence, it is called maximal optimal solution for (DLPI).

The following technical lemma will be useful to prove the theorem. In fact, it reveals that vector $y^{*}$ is the componentwise maximum of all feasible solutions. Recall that by $F$ (DLPI) we denote the feasible solution set for (DLPI).

Lemma 3.2. For each $y \in F(\mathrm{DLPI}), y_{t} \leqslant y_{t}^{*}$ for all $t \in\{1, \ldots, T\}$.

Proof. Take $y \in F(\mathrm{DLPI})$. Let $p_{k_{h}}+h_{k_{h} t}=\min _{k<t}\left\{p_{k}+h_{k t}\right\}$ and $p_{k_{b}}+b_{t k_{b}}=\min _{k>t}\left\{p_{k}+b_{t k}\right\}$, $t=1, \ldots, T$.

For any $t \in\{1, \ldots, T\}$ we can distinguish three situations:

(S1) $p_{t} \leqslant p_{k_{h}}+h_{k_{h} t}$ and $p_{t} \leqslant p_{k_{b}}+h_{k_{b} t}$. Then, $y_{t}^{*}=p_{t}$, and by (8) $y_{t} \leqslant y_{t}^{*}$.

(S2) $p_{k_{h}}+h_{k_{h} t}<p_{t}$ and $p_{k_{h}}+h_{k_{h} t} \leqslant p_{k_{b}}+b_{t k_{b}}$. Then, $y_{t}^{*}=h_{k_{h} t}+p_{k_{h}}$.

Taking into account that

$$
\begin{aligned}
y_{k_{h}} & \leqslant p_{k_{h}}, \\
y_{k_{h}+1} & \leqslant h_{k_{h}}+y_{k_{h}}, \\
y_{k_{h}+2} & \leqslant h_{k_{h}+1}+y_{k_{h}+1} \leqslant h_{k_{h}+1}+\left(h_{k_{h}}+p_{k_{h}}\right)=h_{k_{h} k_{h}+2}+p_{k_{h}}, \\
& \vdots \\
y_{t} & \leqslant h_{t-1}+y_{t-1} \leqslant h_{t-1}+\left(h_{k_{h} t-1}+p_{k_{h}}\right)=h_{k_{h} t}+p_{k_{h}}
\end{aligned}
$$

we conclude that $y_{t} \leqslant y_{t}^{*}$.

$$
\begin{aligned}
p_{k_{b}}+b_{t k_{b}}<p_{t} \text { and } p_{k_{b}}+b_{t k_{b}}<p_{k_{h}}+h_{k_{h} t} . \text { Then, } y_{t}^{*}=b_{t k_{b}}+p_{k_{b}} ; \text { and } \\
y_{k_{b}} \leqslant p_{k_{b}}, \\
y_{k_{b}-1} \leqslant b_{k_{b}-1}+y_{k_{b}}, \\
y_{k_{b}-2} \leqslant b_{k_{b}-2}+y_{k_{b}-1} \leqslant b_{k_{b}-2}+\left(b_{k_{b}-1}+p_{k_{b}}\right)=b_{k_{b}-2 k_{b}}+p_{k_{b}}, \\
\quad \vdots \\
y_{t} \leqslant b_{t}+y_{t+1} \leqslant b_{t}+\left(b_{t+1 k_{b}}+p_{k_{b}}\right)=b_{t k_{b}}+p_{k_{b}} .
\end{aligned}
$$

Hence, $y_{t} \leqslant y_{t}^{*}$.

Theorem 3.3. The optimal solution set of problem (DLPI) is given by

$$
O(\mathrm{DLPI})=\left\{\begin{array}{lll}
y \in F(\mathrm{DLPI}) ; & y_{t} \leqslant y_{t}^{*} & \text { if } d_{t}=0 \\
y_{t}=y_{t}^{*} & \text { if } d_{t}>0
\end{array}\right\} .
$$

Proof. ( $\subseteq$ ) Take $y \in O$ (DLPI). By Lemma 3.2, $y_{t} \leqslant y_{t}^{*}$ for all $t \in\{1, \ldots, T\}$. Suppose there exists a period $t \in\{1, \ldots, T\}$ with $d_{t}>0$ and $y_{t}<y_{t}^{*}$. Then by Lemma 3.1, val(DLPI) $=y_{1}^{*} d_{1}+$ $\cdots+y_{T}^{*} d_{T}>y_{1} d_{1}+\cdots+y_{T} d_{T}$, which is a contradiction. 
() It is obvious that for all $y \in F(\mathrm{DLPI})$ such that $y_{t} \leqslant y_{t}^{*}$, if $d_{t}=0$, and $y_{t}=y_{t}^{*}$ otherwise,

$$
\sum_{t=1}^{T} d_{t} y_{t}=\sum_{\substack{t=1 \\ d_{t}>0}}^{T} d_{t} y_{t}^{*}=\sum_{t=1}^{T} d_{t} y_{t}^{*}
$$

Hence by duality theorem in linear programming, we can conclude that $y \in O$ (DLPI).

\section{Production-inventory games}

Once we have revisited the classical version of the production-inventory problem, we address a natural variant of this problem in which several agents, each one facing a PI-problem, decide to cooperate to reduce costs. Here the cooperation is driven by sharing technologies in production, inventory carrying and backlogged demand. Thus, if a group of agents agree on cooperation then at each period they will produce and pay inventory carrying and backlogged demand at the cheapest costs among the members of the coalition. Cooperation in holding and production costs is usual and has appeared already in literature. Our mode of cooperation in backlogging is also natural: once a coalition is formed, all its members pay compensation to customers for delayed delivering (backlogging cost) of their demands according to the cheapest cost among the members in the coalition. In some regards, larger coalitions are stronger and "squeeze" a bit more their clients. Formally, a production-inventory situation (PI-situation) is a 5-tuple $(N, D, H, B, P)$ where $N$ is the set of players $N=\{1, \ldots, n\}$ and for each player $i$ $\left(T, d^{i}, h^{i}, b^{i}, p^{i}\right)$ is a PI-problem.

The reader may notice that

$$
\begin{array}{ll}
D=\left[d^{1}, \ldots, d^{n}\right]^{\prime}, & H=\left[h^{1}, \ldots, h^{n}\right]^{\prime}, \\
B=\left[b^{1}, \ldots, b^{n}\right]^{\prime}, & P=\left[p^{1}, \ldots, p^{n}\right]^{\prime} ;
\end{array}
$$

and

$$
\begin{aligned}
d^{i} & =\left[d_{1}^{i}, \ldots, d_{T}^{i}\right]^{\prime}, & h^{i} & =\left[h_{1}^{i}, \ldots, h_{T}^{i}\right]^{\prime}, \\
b^{i} & =\left[b_{1}^{i}, \ldots, b_{T}^{i}\right]^{\prime}, & p^{i} & =\left[p_{1}^{i}, \ldots, p_{T}^{i}\right]^{\prime} .
\end{aligned}
$$

Note that we can associate with each PI-situation $(N, D, H, B, P)$ a cost TU-game $(N, c)$ with characteristic function $c$ defined as follows: $c(\emptyset)=0$ and for any $S \subseteq N, c(S)=$ $\operatorname{val}(\operatorname{LPI}(S))$, where

$$
\begin{aligned}
& (\operatorname{LPI}(S)) \quad \min \sum_{t=1}^{T}\left(p_{t}^{S} q_{t}+h_{t}^{S} I_{t}+b_{t}^{S} E_{t}\right) \\
& \text { s.t. } \quad I_{0}=I_{T}=E_{0}=E_{T}=0, \\
& \\
& \quad I_{t}-E_{t}=I_{t-1}-E_{t-1}+q_{t}-d_{t}^{S}, \quad t=1, \ldots, T, \\
& \\
& \quad q_{t} \geqslant 0, I_{t} \geqslant 0, E_{t} \geqslant 0, \quad t=1, \ldots, T ;
\end{aligned}
$$

with

$$
p_{t}^{S}=\min _{i \in S}\left\{p_{t}^{i}\right\}, \quad h_{t}^{S}=\min _{i \in S}\left\{h_{t}^{i}\right\}, \quad b_{t}^{S}=\min _{i \in S}\left\{b_{t}^{i}\right\}, \quad d_{t}^{S}=\sum_{i \in S} d_{t}^{i} .
$$

Every cost TU-game defined in this way is what we call a production-inventory game (PIgame). 
The main result in this section states that every PI-game is totally balanced, hence subadditive.

Theorem 4.1. Let $(N, D, H, B, P)$ be a PI-situation with $D$ being an integer matrix. The corresponding PI-game $(N, c)$ is totally balanced.

Proof. For any $S \subseteq N$ consider the reduced game $\left(S, c_{S}\right)$.

Let $y^{*}$ be an optimal solution of $(\operatorname{DLPI}(S))$. Define $u^{*}=\left(y^{* \prime} d^{i}\right)_{i \in S} \in \mathbb{R}^{s}$. It is clear that

$$
\sum_{i \in S} u_{i}^{*}=\sum_{t=1}^{T} y_{t}^{*} d_{t}^{S}=\operatorname{val}(\operatorname{DLPI}(S))=\operatorname{val}(\operatorname{LPI}(S))=c_{S}(S) .
$$

Moreover, for any $R \subseteq S, y^{*}$ is a feasible solution for (DLPI $\left.(R)\right)$ since the constraint matrix does not depend on $S$ and the right-hand side of $(\operatorname{DLPI}(S))$ is componentwise smaller than or equal to the one of $(\operatorname{DLPI}(R))$. Hence,

$$
c_{S}(R)=\operatorname{val}(\operatorname{DLPI}(R))=\max _{y \in F(\operatorname{DLPI}(R))} \sum_{t=1}^{T} d_{t}^{R} y_{t} \geqslant \sum_{t=1}^{T} d_{t}^{R} y_{t}^{*}=\sum_{i \in R} y^{* \prime} d^{i}=\sum_{i \in R} u_{i}^{*} .
$$

Therefore, $\operatorname{Core}\left(S, c_{S}\right) \neq \emptyset$.

We note in passing that although PI-games are totally balanced games, in general these games are not concave (see Example 4.4).

Recently, Deng et al. (1999, 2000) have studied some families of combinatorial optimization games, namely packing and covering games, for which they prove total balancedness. Here, we have presented a different class exhibiting the same property. The core of the above mentioned classes of combinatorial optimization games coincides with the set of dual solutions, i.e. optimal solutions of the dual problem to the one that defines the characteristic function. However, the same property does not hold in our class of PI-games as will be shown in the next examples. In order to study the core set within the class of PI-games we introduce the so-called Owen set: the set of allocations that are achievable through dual solutions (see Gellekom et al., 2000). Formally, the Owen set of $(N, D, H, B, P)$ is defined by

$$
\operatorname{Owen}(N, D, H, B, P)=\left\{\left(y^{\prime} d^{i}\right)_{i \in N}: y \in O(\operatorname{DLPI}(N))\right\} \text {. }
$$

As a straightforward consequence of Theorem 4.1, we obtain that

$$
\operatorname{Owen}(N, D, H, B, P) \subseteq \operatorname{Core}(N, c) \text {. }
$$

Our next example shows that in general there are core-allocations not achieved with dual solutions.

Example 4.2. Consider the following PI-situation with two periods and two players, namely P1 and P2:

\begin{tabular}{rrrlrlrlr}
\hline & \multicolumn{2}{c}{ Demand } & \multicolumn{2}{c}{ Production } & \multicolumn{2}{c}{ Inventory } & \multicolumn{2}{c}{ Backlogging } \\
\hline P1 & 10 & 10 & 2 & 2 & 1 & 2 & 1 & 2 \\
P2 & 8 & 12 & 1 & 1 & 2 & 4 & 2 & 2 \\
\hline
\end{tabular}

The data above gives rise to the game with characteristic function in the following table. 


\begin{tabular}{lrllllllll}
\hline & $d_{1}^{S}$ & $d_{2}^{S}$ & $p_{1}^{S}$ & $p_{2}^{S}$ & $h_{1}^{S}$ & $h_{2}^{S}$ & $b_{1}^{S}$ & $b_{2}^{S}$ & $c$ \\
\hline$\{1\}$ & 10 & 10 & 2 & 2 & 1 & 2 & 1 & 2 & 40 \\
$\{2\}$ & 8 & 12 & 1 & 1 & 2 & 4 & 2 & 2 & 20 \\
$\{1,2\}$ & 18 & 22 & 1 & 1 & 1 & 2 & 1 & 2 & 40 \\
\hline
\end{tabular}

The unique optimal solution of $(\operatorname{DLPI}(\{1,2\}))$ is $y^{* \prime}=(1,1)$. This gives $\operatorname{Owen}(N, D, H$, $B, P)=\{(20,20)\}$. However, Core $(N, c)=\left\{\left(x_{1}, x_{2}\right): 0 \leqslant x_{1} \leqslant 40,0 \leqslant x_{2} \leqslant 20, x_{1}+x_{2}=40\right\}$.

In order to get the Owen set one has to solve three optimization problems (DLPI $(\{1,2\})$ ), $(\operatorname{DLPI}(\{1\}))$ and $(\operatorname{DLPI}(\{2\}))$. In general, for a $n$-player game $2^{n}-1$ optimization problems would have had to be solved.

For the sake of simplicity, we obtain an explicit form for the characteristic function of PIgames.

Consider $(\operatorname{DLPI}(S))$ the dual of $(\operatorname{LPI}(S))$. Recall that the problem $(\operatorname{DLPI}(S))$ is defined as (DLPI) (see (7)-(10)) where cost coefficients and right-hand side vector are replaced by $p^{S}, h^{S}$ and $b^{S}$, and $d^{S}$, respectively.

Let us denote

$$
\begin{aligned}
& h_{k t}^{S}=\sum_{r=k}^{t-1} h_{r}^{S} \quad \text { for any } k<t, t=2, \ldots, T, h_{k 1}^{S}=0, k<1, \\
& b_{t k}^{S}=\sum_{r=t}^{k-1} b_{r}^{S} \quad \text { for any } k>t, t=1, \ldots, T-1, b_{T k}^{S}=0, k>T .
\end{aligned}
$$

Similar arguments to the ones given in Section 3 show that for all $S \subseteq N, S \neq \emptyset$, the optimal solution set of problem $(\operatorname{DLPI}(S))$ is

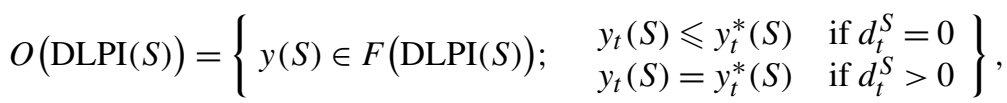

where $y^{*}(S)$, the maximal optimal solution, is now given by

$$
y_{t}^{*}(S)=\min \left\{p_{t}^{S}, \min _{k<t}\left\{p_{k}^{S}+h_{k t}^{S}\right\}, \min _{k>t}\left\{p_{k}^{S}+b_{t k}^{S}\right\}\right\}, \quad t=1, \ldots, T,
$$

with

$$
p_{k}^{S}= \begin{cases}p_{1}^{S} & \text { if } k<1 \\ p_{T}^{S} & \text { if } k>T\end{cases}
$$

Let

$$
p_{k_{h}}^{S}+h_{k_{h} t}^{S}=\min _{k<t}\left\{p_{k}^{S}+h_{k t}^{S}\right\}, \quad p_{k_{b}}^{S}+b_{t k_{b}}^{S}=\min _{k>t}\left\{p_{k}^{S}+b_{t k}^{S}\right\}, \quad t=1, \ldots, T,
$$

and

$$
\begin{aligned}
& H_{1}(S)=\left\{t \in\{1, \ldots, T\} / p_{t}^{S} \leqslant p_{k_{h}}^{S}+h_{k_{h} t}^{S} \text { and } p_{t}^{S} \leqslant p_{k_{b}}^{S}+b_{t k_{b}}^{S}\right\}, \\
& H_{2}(S)=\left\{t \in\{2, \ldots, T\} / p_{k_{h}}^{S}+h_{k_{h} t}^{S}<p_{t}^{S} \text { and } p_{k_{h}}^{S}+h_{k_{h} t}^{S} \leqslant p_{k_{b}}^{S}+b_{t k_{b}}^{S}\right\}, \\
& H_{3}(S)=\left\{t \in\{1, \ldots, T-1\} / p_{k_{b}}^{S}+b_{t k_{b}}^{S}<p_{t}^{S} \text { and } p_{k_{b}}^{S}+b_{t k_{b}}^{S}<p_{k_{h}}^{S}+h_{k_{h} t}^{S}\right\} .
\end{aligned}
$$

Remark that $\left\{H_{1}(S), H_{2}(S), H_{3}(S)\right\}$ is a partition of the planning horizon set $\{1, \ldots, T\}$. According to this notation, it can be checked that an explicit form for the characteristic function of a PI-game is given by (18). 
Proposition 4.3. Let $(N, D, H, B, P)$ be a PI-situation with $D$ being an integer matrix, and $(N, c)$ the corresponding PI-game. Then, for each $S \subseteq N, S \neq \emptyset$

$$
c(S)=\sum_{t \in H_{1}(S)} p_{t}^{S} d_{t}^{S}+\sum_{t \in H_{2}(S)} d_{t}^{S}\left(p_{k_{h}}^{S}+h_{k_{h} t}^{S}\right)+\sum_{t \in H_{3}(S)} d_{t}^{S}\left(p_{k_{b}}^{S}+b_{t k_{b}}^{S}\right) .
$$

Proof. Taking into account that for any $S \subseteq N, c(S)=\operatorname{val}(\operatorname{DLPI}(S))$ and $y^{*}(S) \in O(\operatorname{DLPI}(S))$, we obtain $c(S)=\sum_{t=1}^{T} y_{t}^{*}(S) d_{t}^{S}$. Hence, (18) holds.

The explicit form above turns out to be expectable. It means that if we are in a period in which production costs are less than production-inventory carrying and production-backlogging carrying costs $\left(t \in H_{1}(S)\right)$, we should produce all the demand in that period. On the contrary, if the period is such that production and production-backlogging carrying costs are greater than production-inventory carrying ones $\left(t \in H_{2}(S)\right)$, all the demand should have been produced in the earlier period with minimum cost $\left(k_{h}\right)$ and inventory carried until that period $(t)$. Finally, in a period where production and production-inventory carrying costs are greater than productionbacklogging carrying ones $\left(t \in H_{3}(S)\right)$, all the demand should be produced in the future period with minimum cost $\left(k_{b}\right)$ and backlogging carried until that period $(t)$.

The reader may notice that if the matrices $H, B$ and $P$ are in integer values then the characteristic function of these games is also integer.

The next example illustrates all the results obtained in Section 4.

Example 4.4. Consider the following PI-situation with three periods and three players.

\begin{tabular}{llllllllllllr}
\hline & \multicolumn{3}{c}{ Demand } & \multicolumn{3}{c}{ Production } & \multicolumn{3}{c}{ Inventory } & \multicolumn{3}{c}{ Backlogging } \\
\hline P1 & 0 & 2 & 5 & 3 & 1 & 2 & 2 & 0 & 1 & 1 & 2 & 1 \\
P2 & 0 & 8 & 6 & 4 & 2 & 3 & 2 & 0 & 1 & 1 & 3 & 1 \\
P3 & 0 & 6 & 2 & 3 & 1 & 2 & 2 & 0 & 1 & 1 & 2 & 1 \\
\hline
\end{tabular}

It can be easily checked that for all $S \subseteq N, H_{1}(S)=\{2\}, H_{2}(S)=\{3\}, H_{3}(S)=\{1\}$, and $c(S)=p_{2}^{S} d_{2}^{S}+d_{3}^{S}\left(h_{23}^{S}+p_{2}^{S}\right)+d_{1}^{S}\left(b_{21}^{S}+p_{2}^{S}\right)$. Hence, the data above gives rise to the game with characteristic function in the following table:

\begin{tabular}{llrrlllllllllr}
\hline & $d_{1}^{S}$ & $d_{2}^{S}$ & $d_{3}^{S}$ & $p_{1}^{S}$ & $p_{2}^{S}$ & $p_{3}^{S}$ & $h_{1}^{S}$ & $h_{2}^{S}$ & $h_{3}^{S}$ & $b_{1}^{S}$ & $b_{2}^{S}$ & $b_{3}^{S}$ & $c$ \\
\hline$\{1\}$ & 0 & 2 & 5 & 3 & 1 & 2 & 2 & 0 & 1 & 1 & 2 & 1 & 7 \\
$\{2\}$ & 0 & 8 & 6 & 4 & 2 & 3 & 2 & 0 & 1 & 1 & 3 & 1 & 28 \\
$\{3\}$ & 0 & 6 & 2 & 3 & 1 & 2 & 2 & 0 & 1 & 1 & 2 & 1 & 8 \\
$\{1,2\}$ & 0 & 10 & 11 & 3 & 1 & 2 & 2 & 0 & 1 & 1 & 2 & 1 & 21 \\
$\{1,3\}$ & 0 & 8 & 7 & 3 & 1 & 2 & 2 & 0 & 1 & 1 & 2 & 1 & 15 \\
$\{2,3\}$ & 0 & 14 & 8 & 3 & 1 & 2 & 2 & 0 & 1 & 1 & 2 & 1 & 22 \\
$\{1,2,3\}$ & 0 & 16 & 13 & 3 & 1 & 2 & 2 & 0 & 1 & 1 & 2 & 1 & 29 \\
\hline
\end{tabular}

The optimal solution set of $(\operatorname{DLPI}(\{1,2,3\}))$ is $\left\{\left(y_{1}, 1,1\right) /-1 \leqslant y_{1} \leqslant 2\right\}$. However, the Owen set and the core reduces to a singleton: $\operatorname{Owen}(N, D, H, B, P)=\operatorname{Core}(N, c)=\{(7,14,8)\}$. Moreover, the game above is not concave since $c(\{1,2\})-c(\{2\})=-7<c(N)-c(\{2,3\})=7$. 
Note that even though there are infinite optimal solutions of the problem $(\operatorname{DLPI}(N))$, the Owen set of the PI-situation above reduces to a singleton again. We wonder if every PI-situation exhibits this property. Next section gives an affirmative answer.

\section{Owen point}

It is well known that the larger the player set, the more difficult it is to determine the core of a cost TU-game (i.e. the number of inequalities involved increases rapidly). The goal of this section is to find a core-allocation for a PI-game which is easy to calculate and satisfies good properties.

The first result reveals that the Owen set of a PI-situation shrinks to a singleton and provides an explicit form to compute it.

Theorem 5.1. Let $(N, D, H, B, P)$ be a PI-situation with $D$ being an integer matrix. Then, $\operatorname{Owen}(N, D, H, B, P)=\left\{\left(o_{1}, \ldots, o_{n}\right)\right\}$ where, for each $i \in N$,

$$
o_{i}=\sum_{t \in H_{1}(N)} p_{t}^{N} d_{t}^{i}+\sum_{t \in H_{2}(N)} d_{t}^{i}\left(p_{k_{h}}^{N}+h_{k_{h} t}^{N}\right)+\sum_{t \in H_{3}(N)} d_{t}^{i}\left(p_{k_{b}}^{N}+b_{t k_{b}}^{N}\right) .
$$

Proof. Take $y^{*}(N), y(N) \in O(\operatorname{DLPI}(N)), y^{*}(N) \neq y(N)$. Proving that $\sum_{t=1}^{T} y_{t}(N) d_{t}^{i}=$ $\sum_{t=1}^{T} y_{t}^{*}(N) d_{t}^{i}$, it follows $o_{i}:=\sum_{t=1}^{T} y_{t}^{*}(N) d_{t}^{i}$ which proves (19).

Taking into account that $d_{t}^{N}=0$ if and only if $d_{t}^{i}=0$ for all $i \in N$, and $y_{t}(N)=y_{t}^{*}(N)$ if $d_{t}^{N}>0$

$$
\sum_{t=1}^{T}\left[y_{t}(N)-y_{t}^{*}(N)\right] d_{t}^{i}=\sum_{\substack{1 \leqslant t \leqslant T \\ d_{t}^{N}=0}}\left[y_{t}(N)-y_{t}^{*}(N)\right] d_{t}^{i}+\sum_{\substack{1 \leqslant t \leqslant T \\ d_{t}^{N}>0}}\left[y_{t}(N)-y_{t}^{*}(N)\right] d_{t}^{i}=0 .
$$

Every cost allocation $o=\left(o_{i}\right)_{i \in N}$ of a PI-game defined in this way is what we call the $O$ wen point for $(N, c)$. Obviously, the Owen point is a core-allocation. The Owen point, for each player $i$, can be interpreted as the cost he/she has to pay when producing at the minimum operation cost. Hence, we propose the Owen point as an alternative value for PI-games. We note in passing that if the matrices $P, H$ and $B$ are integers then the core-allocation given by the Owen point is also in integer values.

The Owen point for the situation given in Example 4.4 is obtained by

$$
\begin{aligned}
& o_{1}=p_{2}^{N} d_{2}^{1}+d_{3}^{1}\left(h_{23}^{N}+p_{2}^{N}\right)+d_{1}^{1}\left(b_{21}^{N}+p_{2}^{N}\right)=7, \\
& o_{2}=p_{2}^{N} d_{2}^{2}+d_{3}^{2}\left(h_{23}^{N}+p_{2}^{N}\right)+d_{1}^{2}\left(b_{21}^{N}+p_{2}^{N}\right)=14, \\
& o_{3}=p_{2}^{N} d_{2}^{3}+d_{3}^{3}\left(h_{23}^{N}+p_{2}^{N}\right)+d_{1}^{3}\left(b_{21}^{N}+p_{2}^{N}\right)=8 .
\end{aligned}
$$

Next theorem shows that there is a pmas that realizes the Owen point.

Theorem 5.2. Let $(N, D, H, B, P)$ be a PI-situation with $D$ being an integer matrix, and $(N, c)$ the corresponding PI-game. The Owen point can be reached through a pmas. 
Proof. Define for all $i \in S, S \subseteq N$ and $S \neq \emptyset$,

$$
y_{i}^{S}:=\sum_{t=1}^{T} y_{t}^{*}(S) d_{t}^{i} .
$$

Then for all $S \subseteq N, S \neq \emptyset$

$$
y^{S}(S)=\sum_{i \in S}\left(\sum_{t=1}^{T} y_{t}^{*}(S) d_{t}^{i}\right)=\sum_{t=1}^{T} y_{t}^{*}(S) d_{t}^{S}=c(S),
$$

and for all $S \subseteq R \subseteq N, S, R \neq \emptyset$ and for all $i \in S$,

$$
y_{i}^{S}=\sum_{t=1}^{T} y_{t}^{*}(S) d_{t}^{i} \geqslant \sum_{t=1}^{T} y_{t}^{*}(R) d_{t}^{i}=y_{i}^{R},
$$

since $y_{t}^{*}(S) \geqslant y_{t}^{*}(R)$ (a consequence of $F(\operatorname{DLPI}(R)) \subseteq F(\operatorname{DLPI}(S)$ ) and Lemma 3.2 extended to the coalition $S \subseteq N$ ).

Finally, we see that $y_{i}^{N}=o_{i}$ for all $i \in N$. So, the Owen point $o$ can be reached through the pmas $\left(y^{S}\right)_{\emptyset \neq S \subseteq N}$.

Existence of pmas for the entire class of production-inventory games is important by itself but in addition has an interesting consequence. It allows us to prove that PI-games are strictly included in the class of totally balanced games since in general there are totally balanced games without pmas.

It is also possible to prove the existence of pmas in PI-games using the following analysis: consider a situation with $N$ players and $T$ time periods, but only 1 out of the $|N| \times|T|$ demands is positive. The player with this demand pays the costs, which obviously decreases if the coalition he belongs to increases. This is a natural pmas (this player is some sort of a veto-player). Extending this to a general setting by adding $|N| \times|T|$ of these games provides a pmas in general.

Another observation, from the proof of the above theorem, is that a pmas for any PI-game can be built just taking the Owen point of each subgame and gathering all of them as a collection of vectors.

The Owen point for the game given in Example 4.4 can be reached through the pmas

$$
\left((7)^{\{1\}},(28)^{\{2\}},(8)^{\{3\}},(7,14)^{\{1,2\}},(7,8)^{\{1,3\}},(14,8)^{\{2,3\}},(7,14,8)^{\{1,2,3\}}\right) .
$$

In that example the core is a singleton: the Owen point. This is not a general property for PI-games. Next theorem provides a necessary and sufficient condition for the core of a PI-game to be a singleton. The following concept is required.

Definition 5.3. Let $(N, D, H, B, P)$ be a PI-situation with $D$ being an integer matrix. We say that $i \in N$ is an essential player for $(N, D, H, B, P)$ if there exists at least one period $t \in\{1, \ldots, T\}$ with $d_{t}^{N \backslash\{i\}}>0$ such that $y_{t}^{*}(N)<y_{t}^{*}(N \backslash\{i\})$.

The reader may notice that an essential player is the one for which at least one period exists, in which it is needed by the rest of players in order to produce at a minimum cost a certain demand. On the other hand, an inessential player $i$ is the one which is unnecessary by the grand coalition to operate at minimum cost. 
We denote by $\mathcal{E}$ the set of essential players and by $N \backslash \mathcal{E}$ its complementary set. In the following we prove that the core of a PI-game shrinks to the Owen point just only when all players are inessential for the PI-situation.

Theorem 5.4. Let $(N, D, H, B, P)$ be a PI-situation with $D$ being an integer matrix and $(N, c)$ the corresponding PI-game. Then, $\operatorname{Core}(N, c)=\{o\}$ if and only if $\mathcal{E}=\emptyset$.

Proof. (Only if) Take $(N, D, H, B, P)$ a PI-situation with $D$ being an integer matrix and all players are inessential for it. Then, for all $i \in N, y_{t}^{*}(N \backslash\{i\})=y_{t}^{*}(N)$ for all $t \in\{1, \ldots, T\}$ with $d_{t}^{N \backslash\{i\}}>0$, and $y_{t}^{*}(N \backslash\{i\}) \geqslant y_{t}^{*}(N)$ for all $t \in\{1, \ldots, T\}$ with $d_{t}^{N \backslash\{i\}}=0$. Hence, $c(N \backslash\{i\})=$ $o(N \backslash\{i\})$.

Take $x \in \operatorname{Core}(N, c)$ and recall that $o \in \operatorname{Core}(N, c)$. Then, for all $i \in N, c(N)-c(N \backslash\{i\})=$ $o_{i} \leqslant x_{i}$ and $\sum_{i=1}^{n} x_{i}=\sum_{i=1}^{n} o_{i}$. Hence, $x_{i}=o_{i}$ for all $i \in N$. So we can conclude that $\operatorname{Core}(N, c)=\{o\}$.

(If) Suppose that $\mathcal{E} \neq \emptyset$. Take $j \in \mathcal{E}$, then there exists $t^{*} \in\{1, \ldots, T\}$ with $d_{t^{*}}^{N \backslash\{j\}}>0$ such that $y_{t^{*}}^{*}(N)<y_{t^{*}}^{*}(N \backslash\{j\})$. For any period $t$ satisfying the above condition, there exists $i \in$ $N \backslash\{j\}$ such that $d_{t}^{i}>0$. It can be checked that for all $R \subset N \backslash\{j\}, i \in R$ it holds $y_{t}^{*}(N)<$ $y_{t}^{*}(N \backslash\{j\}) \leqslant y_{t}^{*}(R)$. Hence $o(R)<c(R)$.

Define $\Delta:=\{R \subset N \backslash\{j\}: i \in R\}, 0<\alpha:=\min _{R \in \Delta}\{c(R)-o(R)\}$, and for all $k \in N$

$$
o_{k}^{*}= \begin{cases}o_{k} & k \in N \backslash\{i, j\}, \\ o_{i}+\alpha & k=i, \\ o_{j}-\alpha & k=j .\end{cases}
$$

It can be easily checked that $o^{*} \in \operatorname{Core}(N, c)$. Hence, we can conclude that $\operatorname{Core}(N, c) \neq$ $\{o\}$.

The above proof sheds light onto the structure of the core. If there exists at least one essential player, the rest of players compensate him for cooperation by reducing the cost when producing at minimum production cost according to (20). On the contrary, if no player is essential the unique core-allocation is the one given by the cost generated when producing at minimum production cost.

We would like to conclude this section positioning the Owen point in comparison with other well-known allocations in cooperative game theory. Although due to space requirements we do not include examples in the paper, the interested reader can find examples supporting our claims in the working paper by Guardiola et al. (2004). First of all, in the class of PI-games the Shapley value (Shapley, 1953) is not, in general, a core allocation. Moreover, even in those cases when Shapley value belongs to the core it does not have to coincide with the Owen point. Finally, we point out that the Owen point does not coincide either with the nucleolus (Schmeidler, 1969).

\section{Concluding remarks}

In this paper we have presented a model of cooperation among several firms arising from dynamic production-inventory situations with discrete demand and finite planning horizon. In this model any group of firms can agree to cooperate because fair allocations of operation costs which are stable always exist. In this sense none of the firms would have an incentive to leave the group. From among all the above fair allocations, we propose the Owen point: the allocation 
in which every firm has to pay the minimum cost of operation. This allocation is especially appealing since it can be calculated in polynomial time and, moreover, it can be reached through a pmas.

We note in passing that incorporating selling prices to the considered model would have not modified the structure of the problem, so that all the conclusions would have been the same.

We would like to finish these remarks mentioning some additional topics for further research on the cooperation model considered in this paper:

(1) to find out descriptions of the structure of the core,

(2) axiomatic characterizations of the Owen point,

(3) alternative allocation schemes, and

(4) other forms of cooperation and/or competition, as well as models with concave cost functions.

\section{Acknowledgments}

The research of the authors is partially supported by Spanish Ministry of Science and Technology grants number: BFM2004-0909, HA2003-0121, MTM2005-09184-C02-02, SEC200200112. Authors acknowledge valuable comments made by the guest Editor and the referees.

\section{References}

Anupindi, R., Bassok, Y., Zemel, E., 2001. A general framework for the study of decentralized distribution systems. Manufacturing Service Operations Management 3, 349-368.

Bondareva, O.N., 1963. Some applications of linear programming methods to the theory of cooperative games. Prob. Kibernety 10, 119-139 (in Russian).

Borm, P.E.M., Hamers, H., Hendrickx, R., 2001. Operations research games: A survey. TOP 9, 139-216.

Deng, X., Ibaraki, T., Nagamochi, H., 1999. Algorithmic aspect of the core of combinatorial optimization games. Math. Oper. Res. 24, 751-766.

Deng, X., Ibaraki, T., Nagamochi, H., Zang, W., 2000. Totally balanced combinatorial optimization games. Math. Programm. 87, 441-452.

Dubey, P., Shapley, L.S., 1984. Totally balanced games arising from controlled programming problems. Math. Programm. 29, 245-267.

Eppen, G.D., 1979. Effect of centralization on expected cost in a multi-location newsboy problem. Manage. Sci. 25, 498-501.

Faigle, U., Kern, W., 2000. On the core of ordered submodular cost games. Math. Programm. 87, 483-499.

Garfinkel, R.S., Nemhauser, G.L., 1972. Integer Programming. Wiley, New York.

Gellekom, J.R.G., Potters, J.A.M., Reijnierse, J.H., Engel, M.C., Tijs, S.H., 2000. Characterization of the Owen set of linear production processes. Games Econ. Behav. 32, 139-156.

Gerchak, Y., Gupta, D., 1991. On apportioning costs to customers in centralized continuous review inventory systems. J. Oper. Manage. 10, 546-551.

Granot, D., 1986. A generalized linear production model: A unified model. Math. Programm. 34, $212-222$.

Guardiola, L.A., Meca, A., Puerto, J., 2004. Production-inventory games: A new class of totally balanced combinatorial optimization games. Trabajos de I+D I-2004-8, CIO Universidad Miguel Hernández, Elche.

Hartman, B.C., Dror, M., 1996. Cost allocation in continuous review inventory models. Naval Res. Logist. 43, 549-561.

Hartman, B.C., Dror, M., 2003. Optimizing centralized inventory operations in a cooperative game theory setting. IIE Trans. 35, 243-257.

Hartman, B.C., Dror, M., 2005. Allocation of gains from inventory centralization in newsvendor environments. IIE Trans. 37, 93-107.

Hartman, B.C., Dror, M., Shaked, M., 2000. Cores of inventory centralization games. Games Econ. Behav. 31, $26-49$.

Johnson, L.A., Montgomery, D.C., 1974. Operations Research in Production Planning, Scheduling, and Inventory Control. John Wiley \& Sons, Chichester and New York. 
Kohli, R., Park, H., 1989. A cooperative game model of quantity discounts. Manage. Sci. 35, 693-707.

Meca, A., 2007. A core-allocation family for generalized holding cost games. Math. Methods Oper. Res. In press.

Meca, A., García-Jurado, I., Borm, P.E.M., 2003. Cooperation and competition in inventory games. Math. Methods Oper. Res. 57, 481-493.

Meca, A., Timmer, J., García-Jurado, I., Borm, P.E.M., 2004. Inventory games. Europ. J. Operations Res. 156, $127-139$.

Minner, S., 2007. Bargaining for cooperative economic ordering. Decis. Support Syst. 43, 569-583.

Müller, A., Scarsini, M., Shaked, M., 2002. The newsvendor game has a nonempty core. Games Econ. Behav. 38, 118126.

Robinson, L.W., 1993. Comment on "On apportioning costs to customers in centralized continuous review inventory systems”, by Gerchak and Gupta. J. Oper. Manage. 11, 99-102.

Schmeidler, D., 1969. The nucleolus of a characteristic function game. SIAM J. Appl. Math. 17, 1163-1170.

Shapley, L.S., 1953. A value for $n$-person games. In: Kuhn, H., Tucker, A.W. (Eds.), Contributions to the Theory of Games, vol. II. Princeton Univ. Press, Princeton, NJ, pp. 307-317.

Shapley, L.S., 1967. On balanced sets and cores. Naval Res. Logist. 14, 453-460.

Shapley, L.S., 1971. Cores of convex games. Int. J. Game Theory 1, 11-26.

Shapley, L.S., Shubik, M., 1969. On market games. J. Econ. Theory 1, 9-25.

Shapley, L.S., Shubik, M., 1972. The assignment game. Int. J. Game Theory. 1, 111-130.

Slikker, M., Fransoo, J., Wouters, M., 2005. Cooperation between multiple news-vendors with transshipments. Europ. J. Operations Res. 167, 370-380.

Sprumont, Y., 1990. Population monotonic allocation schemes for cooperative games with transferable utility. Games Econ. Behav. 2, 378-394.

Tamir, A., 1992. On the core of cost allocation games defined on location problems. Transp. Sci. 27, 81-86.

Tijs, S.H., Meca, A., López, M.A., 2005. Benefit sharing in holding situations. Europ. J. Operations Res. 162, $251-269$.

Wagner, H.M., Whitin, T.M., 1958. Dynamic version of the economic lot size model. Manage. Sci. 5, 89-96. 